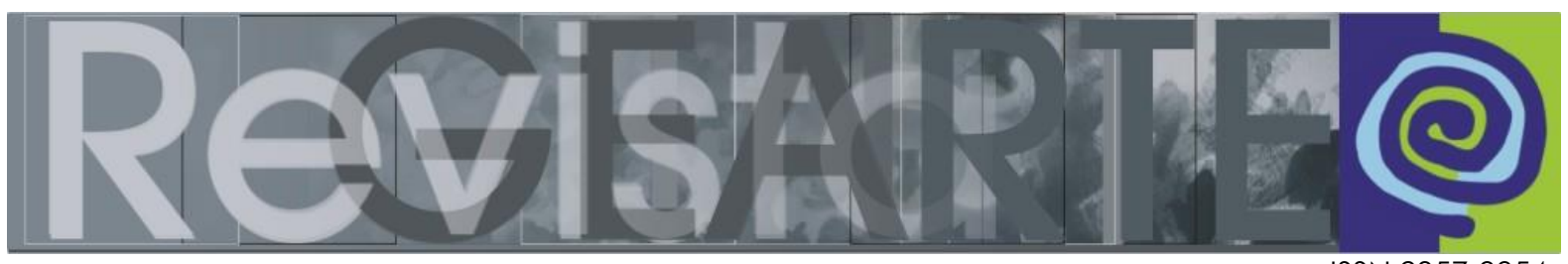

e-ISSN 2357-9854

\title{
Diálogos entre o Ensino de Artes Visuais no Brasil e em outros contextos
}




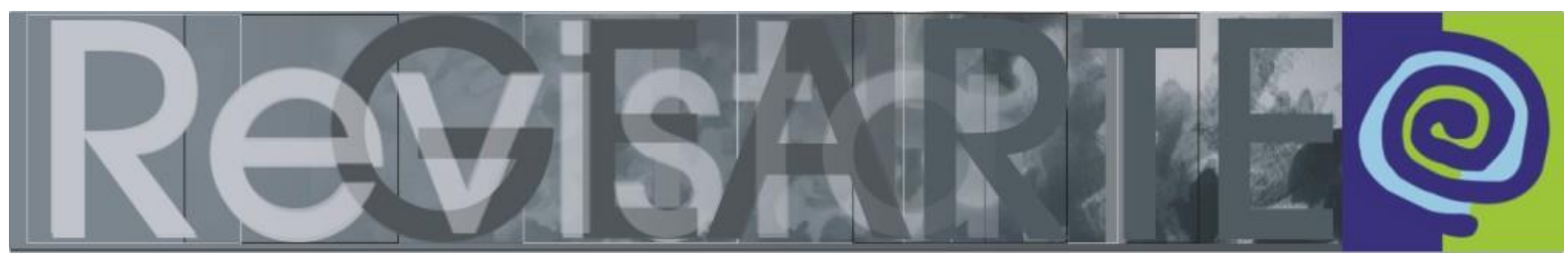

e-ISSN 2357-9854

\title{
EXPEDIENTE
}

A Revista GEARTE é um periódico quadrimestral sob a responsabilidade do Grupo de Pesquisa em Educação e Arte, do Programa de Pós-graduação em Educação da Universidade Federal do Rio Grande do Sul.

\section{Universidade Federal do Rio Grande do Sul \\ Reitor: Rui Vicente Oppermann}

\author{
Faculdade de Educação \\ Diretor: Cesar Valmor Machado Lopes
}

Programa de Pós-graduação em Educação

Coordenador: Luis Henrique Sacchi dos Santos

\section{Editora-Chefe}

Analice Dutra Pillar - Universidade Federal do Rio Grande do Sul (UFRGS), Porto Alegre/RS, Brasil

\section{Editoras Associadas}

Maria Helena Wagner Rossi - Universidade de Caxias do Sul (UCS), Caxias do Sul/RS, Brasil Marília Forgearini Nunes - Universidade Federal do Rio Grande do Sul (UFRGS), Porto Alegre/RS, Brasil Andrea Hofstaetter - Universidade Federal do Rio Grande do Sul (UFRGS), Porto Alegre/RS, Brasil

Flávia Bastos - University of Cincinnati (UC), Cincinnati, Estados Unidos

\section{Editoras Assistentes}

Gabriela Bon - Universidade Federal do Rio Grande do Sul (UFRGS), Porto Alegre/RS, Brasil Tatiana Telch Evalte - Universidade Federal do Rio Grande do Sul (UFRGS), Porto Alegre/RS, Brasil

\section{Conselho Científico Nacional}

Ana Mae Barbosa, Universidade de São Paulo (USP) / Universidade Anhembi Morumbi, São Paulo/SP, Brasil Ana Marta Meira, Universidade Federal do Rio Grande do Sul (UFRGS), Porto Alegre/RS, Brasil Celso Vitelli, Universidade Federal do Rio Grande do Sul (UFRGS), Porto Alegre/RS, Brasil Denise Grinspum, Museus Castro Maya (MCM), Rio de Janeiro / RJ, Brasil

Gilvânia Maurício Dias Pontes, Universidade Federal do Rio Grande do Norte (UFRN), Natal/RN, Brasil Leda Maria de Barros Guimarães, Universidade Federal de Goiás (UFG), Goiânia/ GO, Brasil Lucia Gouvêa Pimentel, Universidade Federal de Minas Gerais (UFMG), Belo Horizonte/MG, Brasil Lucia Teixeira de Siqueira e Oliveira, Universidade Federal Fluminense (UFF), Niterói/RJ, Brasil Lucimar Bello Pereira Frange, Pontifícia Universidade Católica de São Paulo (PUCSP), São Paulo/SP, Brasil Luciana Gruppelli Loponte, Universidade Federal do Rio Grande do Sul (UFRGS), Porto Alegre/RS, Brasil Marcos Villela Pereira, Pontifícia Universidade Católica do Rio Grande do Sul (PUCRS), Porto Alegre/RS, Brasil Maria Isabel Petry Kehrwald, Fundação Municipal de Artes de Montenegro (FUNDARTE), Montenegro/RS, Brasil

Maria Lúcia Batezat Duarte, Universidade do Estado de Santa Catarina (UDESC), Florianópolis/SC, Brasil Marion Divério Faria Pozzi, Universidade Federal do Rio Grande do Sul (UFRGS), Porto Alegre/RS, Brasil Moema Lúcia Martins Rebouças, Universidade Federal do Espírito Santo (UFES), Vitória/ES, Brasil Mirian Celeste Martins, Universidade Presbiteriana Mackenzie (MACKENZIE), São Paulo/SP, Brasil Nadja de Carvalho Lamas, Universidade da Região de Joinville (UNIVILLE), Joinville/SC, Brasil

Regina Maria Varini Mutti, Universidade Federal do Rio Grande do Sul (UFRGS), Porto Alegre/RS, Brasil Rejane Reckziegel Ledur, Universidade Luterana do Brasil (ULBRA), Canoas/RS, Brasil

Rita Inês Petrykowski Peixe, Instituto Federal de Santa Catarina (IFSC Campus de Itajaí), Itajaí/SC, Brasil Rosângela Fachel de Medeiros, Universidade Regional Integrada do Alto Uruguai e das Missões (URI), Frederico Westphalen/RS, Brasil

Sandra Regina Ramalho e Oliveira, Universidade do Estado de Santa Catarina (UDESC), Florianópolis/SC, Brasil Umbelina Duarte Barreto, Universidade Federal do Rio Grande do Sul (UFRGS), Porto Alegre/RS, Brasil 


\section{Conselho Científico Internacional}

Fernando Hernández, Universidad de Barcelona (UB), Barcelona, Espanha Imanol Aguirre Arriaga, Universidad Pública de Navarra, Navarra, Espanha Lourenço Eugênio Cossa, Universidade Pedagógica, Maputo, Moçambique

María Acaso López-Bosch, Universidad Complutense de Madrid (UCM), Madrid, Espanha Michael Parsons, Ohio State University (OSU), Columbus e University of Illinois (UIUC), Urbana-Champaign, Estados Unidos

Norman Freeman, University of Bristol, Bristol, Reino Unido

Raquel Ribeiro dos Santos, Fundação Caixa Geral de Depósito (Culturgest), Lisboa, Portugal Ricardo Marín-Viadel, Universidad de Granada (UGR), Granada, Espanha

Ricardo Rubiales García Jurado, Consejo Estatal para la Cultura y las Artes (CECA), Pachuca, Hidalgo, México Teresa Torres Eça, Universidade do Porto (UP), Porto, Portugal

\section{Revisoras}

Liane Batistela Kist - Centro Universitário Franciscano (UNIFRA), Santa Maria/RS, Brasil Najara Ferrari Pinheiro - Centro Universitário Franciscano (UNIFRA), Santa Maria/RS, Brasil Noara Bolzan Martins - Centro Universitário Franciscano (UNIFRA), Santa Maria/RS, Brasil

\section{Diagramador}

Edson Leonel de Oliveira - Universidade Federal do Rio Grande do Sul (UFRGS), Porto Alegre/RS, Brasil

\section{Bolsista}

Júlia Azambuja dos Santos - Universidade Federal do Rio Grande do Sul (UFRGS), Porto Alegre/RS, Brasil

\section{Capa}

Umbelina Barreto - Universidade Federal do Rio Grande do Sul (UFRGS), Porto Alegre/RS, Brasil

\section{Apoio}

Programa de Apoio à Edição de Periódicos PAEP / UFRGS

\section{Contatos}

Universidade Federal do Rio Grande do Sul

Programa de Pós-Graduação em Educação

Grupo de Pesquisa em Educação e Arte - GEARTE

Av. Paulo Gama, s/no, prédio 12201, sala 727 - Centro, CEP 90046-900, Porto Alegre/RS

Revista: http://www.seer.ufrgs.br/gearte

Site do grupo: http://www.ufrgs.br/gearte

Telefone: (51) 3308-4145

E-mail: gearte.ufrgs@gmail.com 